\title{
BMJ Open Ibobbly mobile health intervention for suicide prevention in Australian Indigenous youth: a pilot randomised controlled trial
}

\author{
Joseph Tighe, ${ }^{1,2}$ Fiona Shand, ${ }^{1}$ Rebecca Ridani, ${ }^{1}$ Andrew Mackinnon, ${ }^{1}$ \\ Nicole De La Mata, ${ }^{3}$ Helen Christensen ${ }^{1}$
}

To cite: Tighe J, Shand F, Ridani $\mathrm{R}$, et al. Ibobbly mobile health intervention for suicide prevention in Australian Indigenous youth: a pilot randomised controlled trial. BMJ Open 2017;7: e013518. doi:10.1136/ bmjopen-2016-013518

- Prepublication history and additional material is available. To view please visit the journal (http://dx.doi.org/ 10.1136/bmjopen-2016013518)

Received 19 July 2016 Revised 2 December 2016 Accepted 6 December 2016

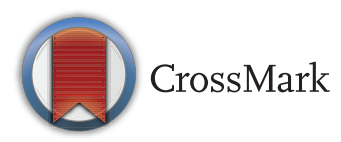

${ }^{1}$ Black Dog Institute, University of New South Wales, Randwick, New South Wales, Australia

${ }^{2}$ Men's Outreach Service Inc., Broome, Western Australia, Australia

${ }^{3}$ Kirby Institute, University of New South Wales,

Kensington, New South

Wales, Australia

Correspondence to Joseph Tighe;

j.tighe@blackdog.org.au

\section{ABSTRACT}

Objectives: Rates of youth suicide in Australian Indigenous communities are 4 times the national youth average and demand innovative interventions. Historical and persistent disadvantage is coupled with multiple barriers to help seeking. Mobile phone applications offer the opportunity to deliver therapeutic interventions directly to individuals in remote communities. The pilot study aimed to evaluate the effectiveness of a self-help mobile app (ibobbly) targeting suicidal ideation, depression, psychological distress and impulsivity among Indigenous youth in remote Australia.

Setting: Remote and very remote communities in the Kimberley region of North Western Australia.

Participants: Indigenous Australians aged 18-35 years. Interventions: 61 participants were recruited and randomised to receive either an app (ibobbly) which delivered acceptance-based therapy over 6 weeks or were waitlisted for 6 weeks and then received the app for the following 6 weeks.

Primary and secondary outcome measures: The primary outcome was the Depressive Symptom Inventory -Suicidality Subscale (DSI-SS) to identify the frequency and intensity of suicidal ideation in the previous weeks. Secondary outcomes were the Patient Health Questionnaire 9 (PHQ-9), The Kessler Psychological Distress Scale (K10) and the Barratt Impulsivity Scale (BIS-11).

Results: Although preintervention and postintervention changes on the (DSI-SS) were significant in the ibobbly $\operatorname{arm}(t=2.40 ; d f=58.1 ; p=0.0195)$, these differences were not significant compared with the waitlist arm ( $\mathrm{t}=1.05$; $d f=57.8 ; p=0.2962)$. However, participants in the ibobbly group showed substantial and statistically significant reductions in PHQ-9 and K10 scores compared with waitlist. No differences were observed in impulsivity. Waitlist participants improved after 6 weeks of app use. Conclusions: Apps for suicide prevention reduce distress and depression but do not show significant reductions on suicide ideation or impulsivity. A feasible and acceptable means of lowering symptoms for mental health disorders in remote communities is via appropriately designed self-help apps.

Trial registration number: ACTRN12613000104752.
Strengths and limitations of this study

- This trial ran successfully in eight distinct Indigenous communities through collaboration between a non-Indigenous research organisation and local Indigenous stakeholders.

- This is the first randomised controlled trial using an app for suicide prevention anywhere including Indigenous Australian communities.

- There was high adherence to the intervention and a very low attrition rate $(3 \%)$ indicating the feasibility of this type of intervention in Indigenous Australian communities.

- The study had a small sample size ( $\mathrm{N}=61)$.

- The study included 16 participants without suicidal ideation at baseline thereby reducing the possibility of achieving significant results in suicidal ideation.

\section{INTRODUCTION}

Suicide rates in Australia for Indigenous communities are twice that of the non-Indigenous population ${ }^{12}$ and four times the rate for youth. ${ }^{2}$ Indigenous suicide differs from non-Indigenous not least in terms of the different historical contexts, ${ }^{3}{ }^{4}$ the broader sociocultural and sociopolitical issues, ${ }^{34}$ the triggers for suicidal behaviour, ${ }^{45}$ the suicidal behaviours themselves, ${ }^{4}{ }^{5}$ and the conceptualisation of suicide within the context of social and emotional well-being (figure 1). ${ }^{4}$ In some communities threats of suicide are often made by both children and adults during interpersonal conflicts, and tragically many threats end in a suicide. ${ }^{5}$ The social determinants of Indigenous health have their origins in dispossession and are

${ }^{\mathrm{i}}$ The term Indigenous will be used throughout to refer to Australian peoples of Aboriginal or Torres Strait Islander descent. Note that most suicide prevention research in Australia is carried out within Aboriginal communities rather than the Torres Strait. 


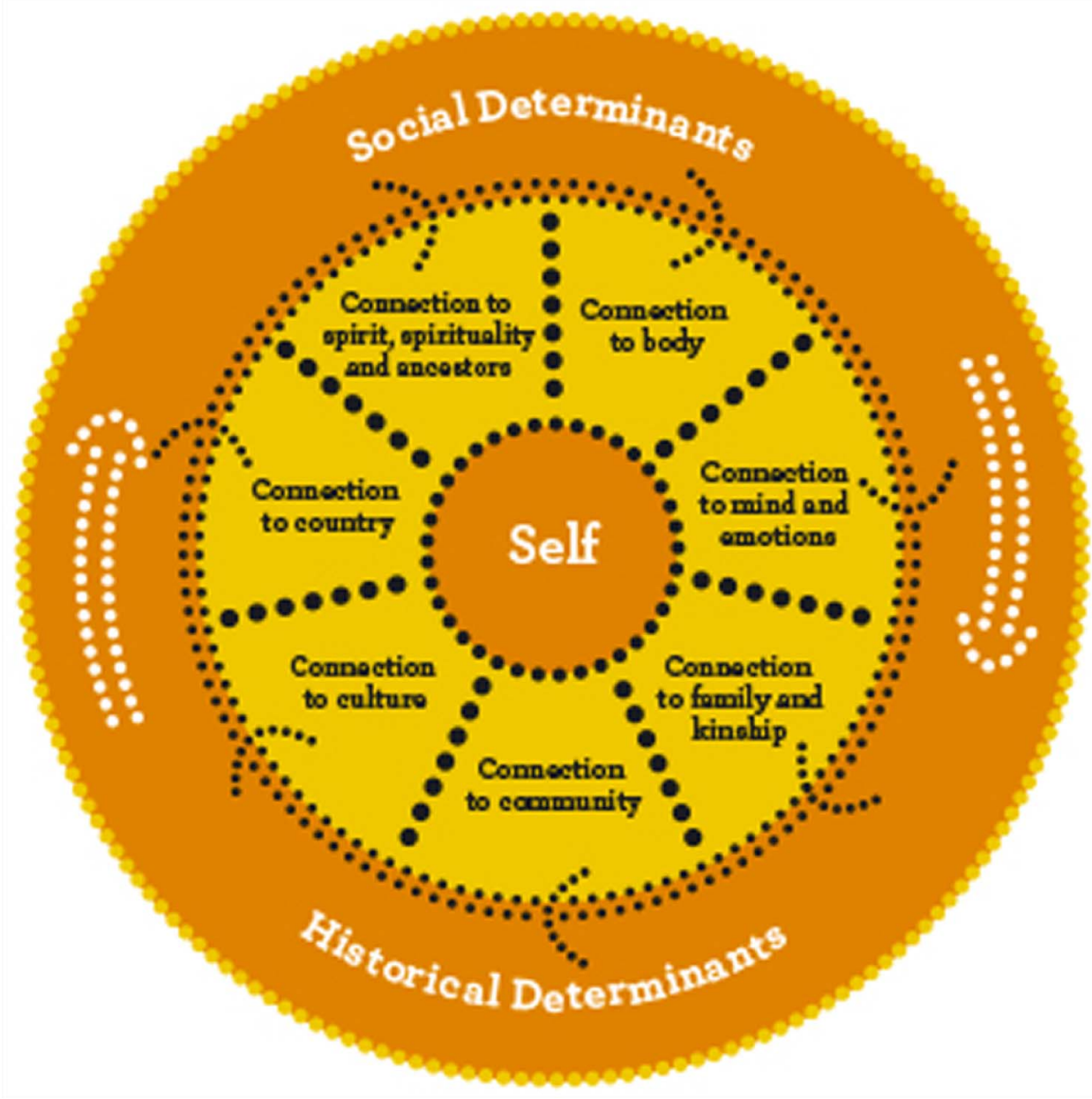

Figure 1 Determinants of social and emotional well-being. ${ }^{6}$

characterised by poverty and powerlessness. ${ }^{1}{ }^{7}$ This context impacts on the help-seeking behaviours of Indigenous Australians. Often mental health services do not exist in remote areas, and when they do, they are not routinely deemed trustworthy or culturally competent. ${ }^{8}$ Barriers to help seeking for mental health issues include remoteness, a lack of appropriate services, mistrust of mainstream services due to negative experiences such as racism, and shame. ${ }^{8}$ Consequently there is significantly less help-seeking behaviour among the Indigenous population compared with other Australians. ${ }^{8}$ More than half of Indigenous people in a 2012-2103 survey reported that a poor opinion of or dislike of the health services prevented them from seeking mental health counselling. ${ }^{9}$ A South Australian study reported that only $14 \%$ of Indigenous people with a diagnosed mental illness at the time of their suicide sought treatment for their illness. ${ }^{10}$

In a space often regarded as 'too difficult' to make progress, opportunities are nevertheless present and the need for innovation is clear. Indigenous-driven suicide prevention and well-being efforts are reporting good outcomes $^{11-13}$ although the potential of technological interventions for this high-risk population has remained largely unexplored. Seventy per cent of Indigenous people own a smart phone compared with $66 \%$ of the overall Australian population, with Indigenous youth accessing social media such as Facebook almost $20 \%$ more frequently than other Australian youth. ${ }^{14}$ Mobile and eHealth technologies may help overcome confidentiality fears, while providing evidence-based therapeutic content on demand in areas without suitable mental health services.

Based on acceptance-based therapies including acceptance and commitment therapy, ${ }^{15}$ the ibobbly app-delivered on an Android tablet-was developed by the Black Dog Institute in partnership with Indigenous community members from the Kimberley, Western Australia. Indigenous artists and graphic designers created original imagery to represent the key messages and activities of the therapeutic content. Full gender-matched audio was developed to overcome low literacy and to follow cultural protocol. The trial aimed to test the app's impact on suicidal ideation, depression, psychological distress and impulsivity, and to pilot the implementation of a randomised controlled trial (RCT) in Indigenous communities focused on suicide prevention. 


\section{METHODS}

\section{Study design}

A two-arm RCT (one intervention group and a 6-week waitlist control group) was conducted, with post-test, and 6-week-follow-up. Recruitment started in September 2013 and ended in March 2015. The study protocol has been published. ${ }^{16}$

\section{Participants}

Recruitment took place through collaboration with a partner organisation Men's Outreach Service (MOS) in Broome, Western Australia. MOS have managed the Alive and Kicking Goals (AKG) ${ }^{17}$ Indigenous youth suicide prevention project since 2008 in partnership with Indigenous community volunteers. The recruitment strategy included a dedicated Facebook page, posters and flyers placed in medical centres, flyers emailed to health professionals, and information sessions held with health professionals. The recruitment also relied on the promotion of the research by Indigenous staff to their networks through word-of-mouth. Initially, participants were eligible if aged between 18 and 35 years of age, had a score of more than 10 on the Patient Health Questionnaire 9 (PHQ-9) or a score of 25 or greater score on the Kessler Psychological Distress Scale (K10) and had suicidal thoughts in the previous 2 weeks.

During the screening process, individuals were asked five questions relating to active suicidality over the previous 2 weeks, including ideation, attempts and current intent. They were also asked if they had a diagnosis of schizophrenia or any other psychotic disorder. Individuals who had active intent to suicide, or had experienced schizophrenia or psychosis were excluded. All screening was completed by the research officer/ psychologist at AKG, with the exception of one individual who was screened by the research assistant at the Black Dog Institute via telephone (participant's preference). Initial screening typically took up to $30 \mathrm{~min}$ with a further $30 \mathrm{~min}$ required to register the individual if eligible.

Changes to the eligibility criteria from the protocol occurred in the first weeks of the trial. These were prompted by feasibility (ethics were approved for only one region rather than nationally thereby significantly reducing the number of potential participants), by local cultural issues, high rates of distress of those above the age limit but wanting to join the trial and restricted health services for this target group. Sixteen participants without recent suicidal ideation but meeting all other criteria were included $(n=16)$ in the trial from 19/09/ 2013, shortly after trial initiation. Seven participants older than 35 years with high distress and high motivation to be included were also included from 19/09/ 2013.

\section{Procedure}

Following screening, eligible participants $(\mathrm{N}=61)$ were randomised (1:1) either to the intervention or waitlist control group using block randomisation stratified by gender (16 per block), using computer-generated randomisation. Each block randomisation was performed offline by a member of the research team at the Black Dog Institute and sent to the research officer in Broome who was responsible for and not blind to the intervention allocation. Participants received the intervention for 6 weeks or joined a 6 -week waitlist. The waitlist control group also received the ibobbly programme 6 weeks after baseline questionnaires were completed. Before starting the programme, all participants received a 15 min training session on how to operate the tablet and app which included setting a password for secure access. Measures were taken face-to-face at baseline and at the beginning and end of the intervention for both groups. In addition to providing helpline and service provider information, safety checks to assess for active suicidality were conducted via telephone at the 3-week and 9-week time points. All participants had face-to-face or telephone contact with the research officer/psychologist at baseline assessment, 3 weeks (safety check) and 6 weeks follow-up. Waitlist group participants also received a further safety check at 9 weeks, and final follow-up assessment at 12 weeks.

\section{Intervention}

The ibobbly programme was delivered via a passwordprotected app provided at no cost to participants. The app contained three content modules and three selfassessments, to be completed over 6 weeks. Participants were expected to progress unprompted through the content. All activities were required to be completed in sequence. Repetition of activities was encouraged to improve learning and impact. Live mobile network connectivity was not necessary to use the app, with online data downloaded once individuals entered internetenabled areas.

In addition to the three main modules, the app required participants to regularly complete selfassessments on their functioning and state of mind, including suicidality. Participants could track their progress through a personalised dashboard. To cater for low literacy levels, all text was accompanied by matching voice recordings. The app displayed emergency contact information for several 24-hour helplines. In module 1, participants learnt to identify thoughts (including suicidal thoughts), feelings and behaviours (including suicidal behaviour), and learnt distancing techniques. In module 2, participants were taught to regulate their emotions through (1) mindfulness, which encouraged participants to maintain non-judgemental contact with their psychological and environmental experiences; (2) acceptance, which presented participants with an alternative to experiential avoidance and helped them to increase their awareness of their internal experiences without the need to control them; and (3) self-soothing activities that were social (eg, calling a friend), active (eg, going for a walk) and cultural (eg, storytelling). 
In module 3, participants were helped to identify values important to them and asked to set small, achievable goals to help them live by these values, and at the end, provided with a personalised action plan, based on prior answers. The suicide-specific content is outlined in online supplementary appendix A.

\section{Measures}

Primary outcome: suicidal ideation (DSI-SS)

The Depressive Symptom Inventory-Suicidality Subscale (DSI-SS) is a four-item self-report questionnaire designed to identify the frequency and intensity of suicidal ideation in the previous weeks. ${ }^{18}$ Total inventory scores range from 0 to 12 , with each answer being scored $0-3$, and higher scores indicating higher severity. The four questions assess frequency of suicidal ideation, development of a suicide plan, an inability to control suicidal thoughts, and suicidal impulses. The scale has good reliability and validity ${ }^{19} 20$ specifically among a group of 15-24 years old general practice patients. ${ }^{21}$

\section{Secondary outcome measures}

Depression: The PHQ-9 consists of nine items, scored from 0 (not at all) to 3 (nearly every day) and rated over previous fortnight. Overall scores range from 0 to 27 with 0-4 representing no or minimal depression, 5-9 representing mild depression, 10-14 moderate depression, 15-19 moderately severe depression and 20-27 severe depression. ${ }^{22}$ For analyses of cases, participants with scores on the PHQ-9 of 10 and above were classified as likely to meet criteria for moderate or more severe depression.

Psychological distress: K10 contains 10 items rated on their occurrence over the previous 4 weeks from 1 (none of the time) to 5 (all of the time). Total scores range from 10 to 50 with psychological distress being categorised as follows: 1-20 are likely to be well; 21-24 are likely to have a mild mental disorder; 25-29 are likely to have a moderately severe mental disorder; 30 and above are likely to have a severe mental disorder. ${ }^{23}$ Participants with K10 scores of 25 or greater were classified as having moderate or more severe psychological distress. The cut-off points used in these analyses were developed for the Australian National Survey of Mental Health and Wellbeing. ${ }^{24}$ There is little evidence regarding the applicability of these classifications for the current sample. It is nevertheless widely used among the Australian Indigenous population.

Impulsivity: The Barratt Impulsivity Scale (BIS-11) is a 30-item questionnaire which asks respondents to select how frequently they engage in common impulsive and non-impulsive behaviour. Responses are coded on a fourpoint scale from 1 (rarely or never) to 4 (almost always or always), with higher scores indicating greater impulsivity. The scale has good validity with internal consistency coefficients ranging from 0.79 to 0.83 and has been used with undergraduate students, substance abuse patients, prison inmates and general psychiatric patients. ${ }^{25}$

\section{Use of health services}

Data were collected at baseline regarding participant's use of health and social care services over the previous 6 months. The Client Service Receipt Inventory $(\mathrm{CSRI})^{26}$ is used in mental health service evaluations and collects retrospective information about the use of health and social care services, accommodation, and income. The following domains were captured within this study: general practitioner consultations, outpatient visits, use of hospital services for physical or mental health problems, mental health helpline contacts, psychiatric crisis support team contacts, social worker contacts, counselling contacts, therapy contacts, self-help groups' contact, and psychiatrist contacts.

\section{Statistical analysis}

Power analysis for the primary outcome measure indicated that 98 participants were needed to attain power of $80 \%$ for a medium-sized clinical effect (difference between arms of change from preintervention to postintervention of $0.75 \mathrm{SDs})$. Analyses of trial outcomes were undertaken on an intent-to-treat basis using a used mixed-model repeated measures analysis of variance framework with factors of treatment arm (activeibobbly vs comparator-waitlist) and occasion of measurement (preintervention, postintervention and 6-week follow-up). This includes all available data for participants and yields unbiased estimates of intervention effects in the assumption that data were missing at random. Follow-up data were available for waitlist participants following their use of the ibobbly intervention subsequent to postintervention assessments. The effectiveness of the intervention was assessed by a planned contrast comparing preintervention and postintervention changes between arms. For descriptive purposes, an additional contrast assessed change in the waitlist condition after these participants had access to the ibobbly app. Within-participant covariation was accommodated using unconstrained variance-covariance matrices and estimated degrees of freedom (df) using Satterthwaite's correction. Effects were calculated from observed data postintervention using pooled estimates of the SD. SPSS V.23 was used for all analyses.

Role of funding source. The Australian Government Department of Health and Ageing provided initial funds for the project, but had no input into design or outcomes.

\section{RESULTS}

Sixty-five participants were screened between September 2013 and December 2014. Two did not meet inclusion criteria (one was diagnosed with schizophrenia and one had scores below cut-off). The remaining two were eligible but chose not to participate (figure 2). The 


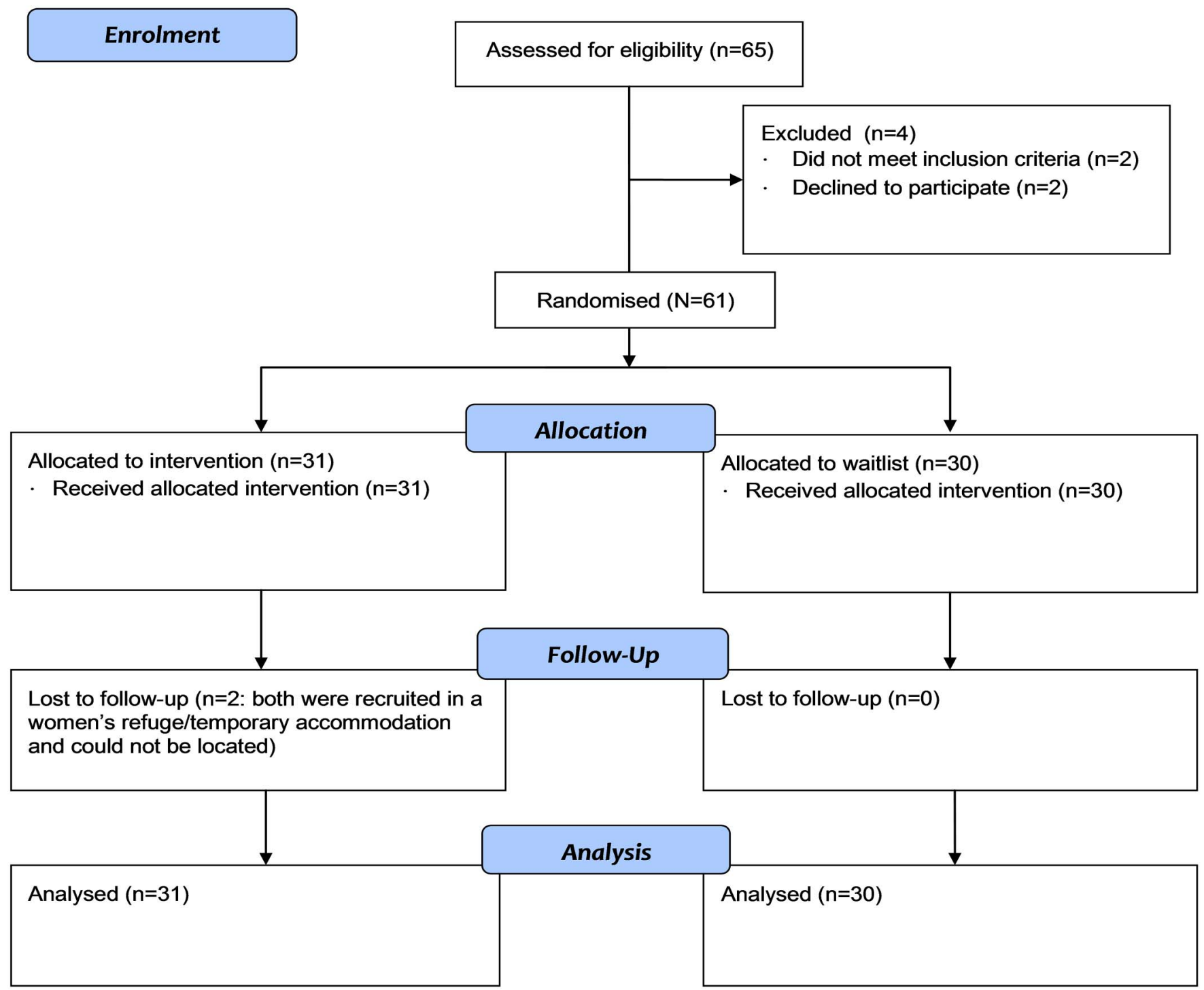

Figure 2 Trial flow diagram.

majority $(75 \%)$ of referrals originated from Indigenous health professionals through word-of-mouth. Mainstream mental health services provided the remaining $25 \%$ of referrals.

Table 1 provides the demographic and health data. While the recruitment took place in the Kimberley region, participants were residing in eight distinct communities namely Broome (remote), Derby (very remote), Fitzroy Crossing (very remote), Djarindjin (very remote), Lombadina (very remote), Mowanjum (very remote), Wangkatjungka (very remote) and Ringer's Soak (very remote).

At baseline $56 \%$ of participants reported receipt of a mental health service in the previous 6 months $(n=34)$. A number of participants $(n=10)$ had received mental health treatment in a hospital setting. However, this does not necessarily indicate a crisis or suicide attempt. In remote communities, the hospital may have been the only possible pathway to care, or the most familiar.
A smaller percentage $(34 \%)$ received general medical care over the previous 6 months. Baseline characteristics, including level of employment, education, gender and Indigenous status, were similar between ibobbly and waitlist participants. Observed means and SDs for all outcome variables at each time point are presented in table 2.

\section{Outcomes}

Suicidal ideation (DSI-SS)

Although preintervention and postintervention changes were significant in the ibobbly $\operatorname{arm}(\mathrm{t}=2.40 ; \mathrm{df}=58.1$; $\mathrm{p}=0.0195$ ), the interaction of intervention arm by time (preintervention vs postintervention) was not significant $(\mathrm{t}=1.05 ; \mathrm{df}=57.8 ; \mathrm{p}=0.2962)$. Estimated marginal means in figure 3 show that any difference between change in the two arms arose because of a slight but nonsignificant difference $(\mathrm{t}=0.84 ; \mathrm{df}=59.0 ; \mathrm{p}=0.3998)$ in mean baseline status between the two arms. Observed 
Table 1 Descriptive statistics

\begin{tabular}{|c|c|c|c|}
\hline Characteristics & $\begin{array}{l}\text { Waitlist ( } n=30) \\
\text { Frequency (per cent) }\end{array}$ & $\begin{array}{l}\text { Intervention }(n=31) \\
\text { Frequency (per cent) }\end{array}$ & Total $(\mathrm{N}=61)$ \\
\hline \multicolumn{4}{|l|}{ Gender } \\
\hline Male (\%) & $11(37)$ & $11(35)$ & \\
\hline Age, mean (SD) & $24.97(6.28)$ & $27.48(9.54)$ & $26.25(8.13)$ \\
\hline Age range (youngest to oldest) & & & $18-56$ \\
\hline \multicolumn{4}{|l|}{ Aboriginal or Torres Strait Islander origin, $\mathrm{n}(\%)$} \\
\hline Aboriginal & $25(83)$ & $30(97)$ & $55(90)$ \\
\hline Torres Strait Islander & 0 & $1(3)$ & $1(2)$ \\
\hline Both aboriginal and Torres Strait Islander & $1(3)$ & 0 & $1(2)$ \\
\hline Neither aboriginal or Torres Strait Islander & $4(13)$ & 0 & $4(6.6)$ \\
\hline \multicolumn{4}{|l|}{ Relationship situation, $\mathrm{n}(\%)$} \\
\hline Married & 0 & $1(3)$ & $1(2)$ \\
\hline De facto & $10(33)$ & $14(45)$ & $24(39)$ \\
\hline Separated & $3(10)$ & $6(19)$ & $9(15)$ \\
\hline \multicolumn{4}{|l|}{ Employment and education outcomes } \\
\hline \multicolumn{4}{|l|}{ Employment status, $n(\%)$} \\
\hline Employed full-time & $9(30)$ & $10(32)$ & $19(31)$ \\
\hline Employed part-time & $4(13)$ & $5(16)$ & $9(15)$ \\
\hline Unemployed-looking for work & $12(40)$ & $11(35)$ & $23(38)$ \\
\hline Not in the labour force & $3(10)$ & $5(16)$ & $8(13)$ \\
\hline \multicolumn{4}{|l|}{ Main activity (if not in the labour force), $n(\%)$} \\
\hline Home duties/caring for children & $9(30)$ & $12(39)$ & $21(43)$ \\
\hline \multicolumn{4}{|l|}{ Schooling level completed, n (\%) } \\
\hline Some secondary & 0 & $5(16)$ & $5(8)$ \\
\hline Four years of secondary & $11(37)$ & $17(55)$ & $28(46)$ \\
\hline Six years of secondary & $19(63)$ & $9(29)$ & $28(46)$ \\
\hline \multicolumn{4}{|l|}{ Tertiary schooling, n (\%) } \\
\hline None & $9(30)$ & $11(35)$ & $20(33)$ \\
\hline Trade/apprenticeship & $3(10)$ & $3(10)$ & $6(10)$ \\
\hline Other certificate & $18(60)$ & $13(42)$ & $31(51)$ \\
\hline Bachelor's degree & 0 & $3(10)$ & $3(5)$ \\
\hline \multicolumn{4}{|l|}{ Current study, n (\%) } \\
\hline None & $23(77)$ & $25(81)$ & $48(79)$ \\
\hline
\end{tabular}

postintervention means were identical leading to an estimated effect size (Cohen's d) of 0.00 (95\% CI -0.51 to $0 \cdot 51)$.

DSI-SS scores of participants in the waitlist arm continued to decline when they were offered access to ibobbly. However, the extent of change was less than in the initial preintervention and postintervention phases, although not significantly so $(\mathrm{t}=-0.29 ; \mathrm{df}=28.9 ; \mathrm{p}=0.7741)$.

\section{Depression (PHQ-9)}

As shown in figure 4, participants in the ibobbly arm showed a substantial and statistically significant reduction in PHQ-9 scores compared with waitlist controls. The interaction of intervention arm by time (preintervention vs postintervention) was significant $(\mathrm{t}=2.79$; $\mathrm{df}=56.9 ; \mathrm{p}=0.0072)$. Cohen's $\mathrm{d}$ was $0.71(95 \%$ CI 0.17 to 1.23), reflecting a substantial effect.

PHQ-9 scores of participants in the waitlist arm continued to decline when they were offered access to ibobbly; however, the extent of change was less than in the initial preintervention and postintervention phases, although not significantly so $(\mathrm{t}=-0.24 ; \mathrm{df}=34.7 ; \mathrm{p}=0.8086)$.

\section{Depression caseness}

Participants with PHQ-9 scores of 10 or greater were classified as likely to meet criteria for moderate or more severe depressive disorder. Only three participants in each arm fell below this cut-off preintervention, so that $90 \%$ of the sample $(55 / 61)$ were likely to be depressed. Postintervention, $58 \%(21 / 30)$ of waitlist participants meet the criteria for depression while only $42 \%(15 / 31)$ of those in ibobbly arm did so. This difference was not statistically significant (Fisher's exact test $\mathrm{p}=0.1196$ ). This corresponds to a relative risk of being depressed after accessing ibobbly of 0.69 (95\% CI 0.45 to 1.06 ) and a number needed to treat of $4.6(95 \%$ CI 2.2 to $\infty$ to $\left.41.0^{\mathrm{ii}}\right)$.

\section{General psychological distress (K10)}

As shown in figure 5, participants in the ibobbly arm also showed a substantial and statistically significant

${ }^{\text {ii }} \mathrm{CI}$ passes through infinity so that number needed to treat is greater than 2.2 and number needed to harm is greater than 41.0. 
Table 2 Observed mean scores for outcomes at: T1 (baseline), T2 (end of 6-week intervention for ibobbly group and start of intervention for waitlist group), T3 (end of intervention for waitlist group)

\begin{tabular}{|c|c|c|c|}
\hline $\begin{array}{l}\text { Outcome } \\
\text { measures }\end{array}$ & $\begin{array}{l}\text { T1 } \\
\text { (baseline) } \\
\text { Mean (SD) }\end{array}$ & $\begin{array}{l}\text { T2 } \\
\text { (6 weeks) } \\
\text { Mean (SD) }\end{array}$ & $\begin{array}{l}\text { T3 } \\
\text { (12 weeks) } \\
\text { Mean (SD) }\end{array}$ \\
\hline \multicolumn{4}{|c|}{ Suicidal ideation } \\
\hline Intervention & $2.7(2.2)$ & $1.9(2.1)$ & \\
\hline Control & $2.3(1.9)$ & $1.9(1.7)$ & $1.5(1.8)$ \\
\hline \multicolumn{4}{|l|}{ Depression } \\
\hline Intervention & $15.2(4.5)$ & $8.9(5.4)^{\star \star}$ & \\
\hline Control & $15.1(3.9)$ & $12.8(5.5)$ & $10.8(5.4)$ \\
\hline \multicolumn{4}{|c|}{ Psychological distress } \\
\hline Intervention & $31.6(5.7)$ & $22.7(7.4)^{\star}$ & \\
\hline Control & $31.6(6.0)$ & $27.9(8.0)$ & $26.2(6.9)$ \\
\hline \multicolumn{4}{|l|}{ Impulsivity } \\
\hline Intervention & $72.1(8.6)$ & $70.6(10.6)$ & \\
\hline Control & $67.9(7.7)$ & $70.6(10.4)$ & $68.1(9.0)$ \\
\hline
\end{tabular}

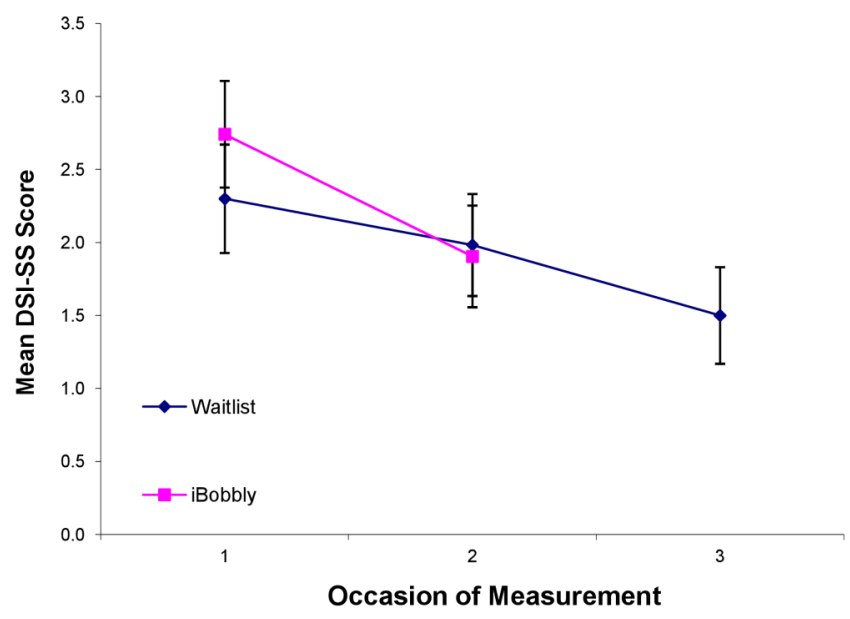

Figure 3 Estimated marginal mean suicide ideation (DSI-SS) scores by time and intervention arm (error bars represent $\pm 1 \mathrm{SE}$ ). DSI-SS, Depressive Symptom InventorySuicidality Subscale.

reduction in K10 scores compared with waitlist controls. The interaction of intervention arm by time (preintervention vs postintervention) was significant $(\mathrm{t}=2.44$; $\mathrm{df}=57.5 ; \mathrm{p}=0.0177)$. Cohen's $\mathrm{d}$ was 0.65 (95\% CI 0.12 to 1.17), reflecting a substantial effect. There was little further decline in K10 scores of participants in the waitlist arm when they were offered access to ibobbly. This reduction was not significant $(\mathrm{t}=-0.24 ; \mathrm{df}=34.7$; $\mathrm{p}=0.8086$ ).

\section{General distress caseness}

Participants with K10 scores of 22 or greater were classified as having high levels of psychological distress. Only three participants fell below this cut-off preintervention,

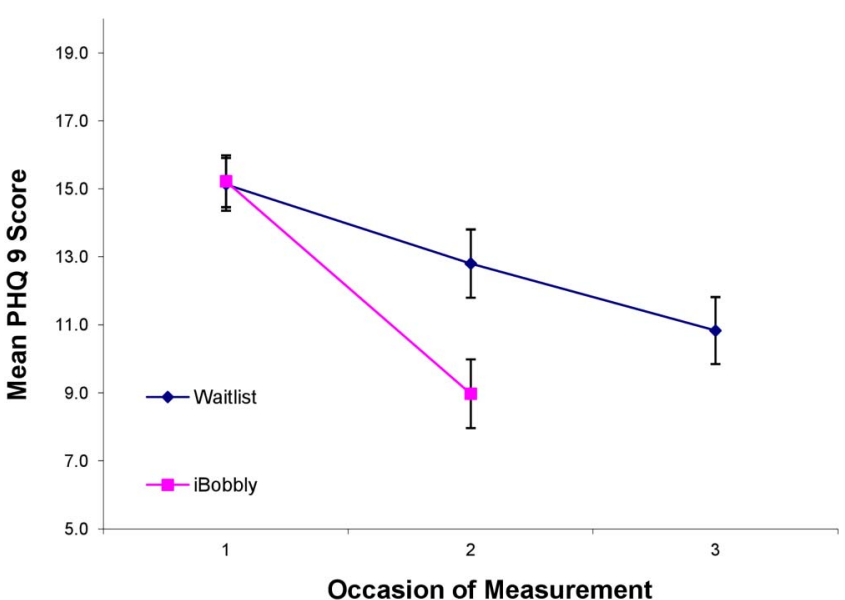

Figure 4 Estimated marginal mean depression (PHQ-9) scores by time and intervention arm (error bars represent \pm 1 SE). PHQ-9, Patient Health Questionnaire 9.

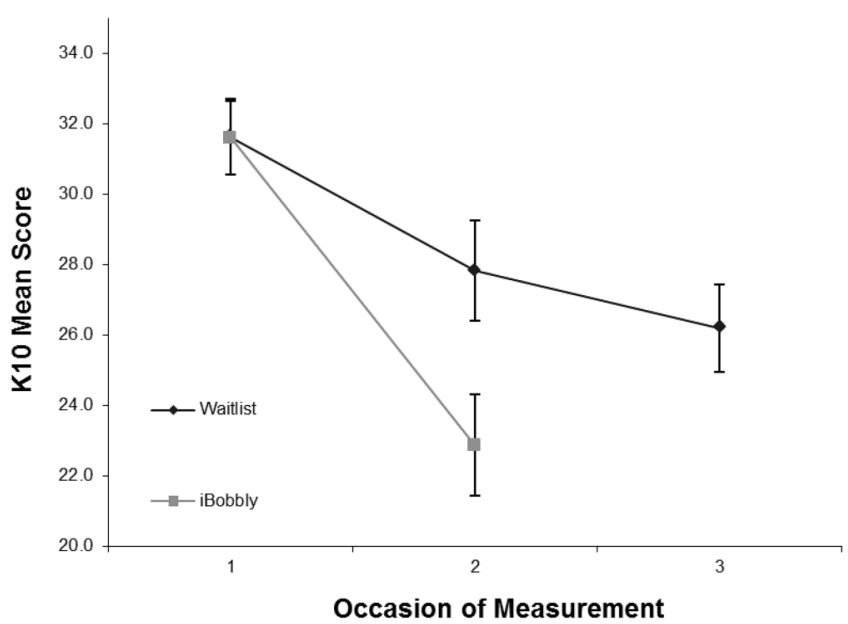

Figure 5 Estimated marginal mean psychological distress (K10) scores by time and intervention arm (error bars represent \pm 1 SE). K10, Kessler Psychological Distress Scale.

so that $95 \%$ of the sample $(58 / 61)$ were classified as highly distressed before the intervention. Postintervention, 59\% (22/29) of waitlist participants had high levels of distress while only $40 \%(15 / 29)$ of those in ibobbly arm did so. This difference was not statistically significant (Fisher's exact test $\mathrm{p}=0.1001$ ). This corresponds to a relative risk of being depressed after accessing ibobbly of 0.68 (95\% CI 0.45 to 1.02 ) and a number needed to treat of 4.1 (95\% CI 2.1 to 516.4$)$.

\section{Impulsivity (BIS-11)}

As shown in figure 6, preintervention scores on the BIS-11 for the waitlist group were significantly lower than for the ibobbly group $(\mathrm{t}=2.05 ; \mathrm{df}=59.2 ; \mathrm{p}=0.0446)$. Postintervention means were identical. Scores in the waitlist decreased from postintervention to follow-up although this was not significant $(\mathrm{t}=-1.82 ; \mathrm{df}=29.1$; $\mathrm{p}=0.0792$ ). 


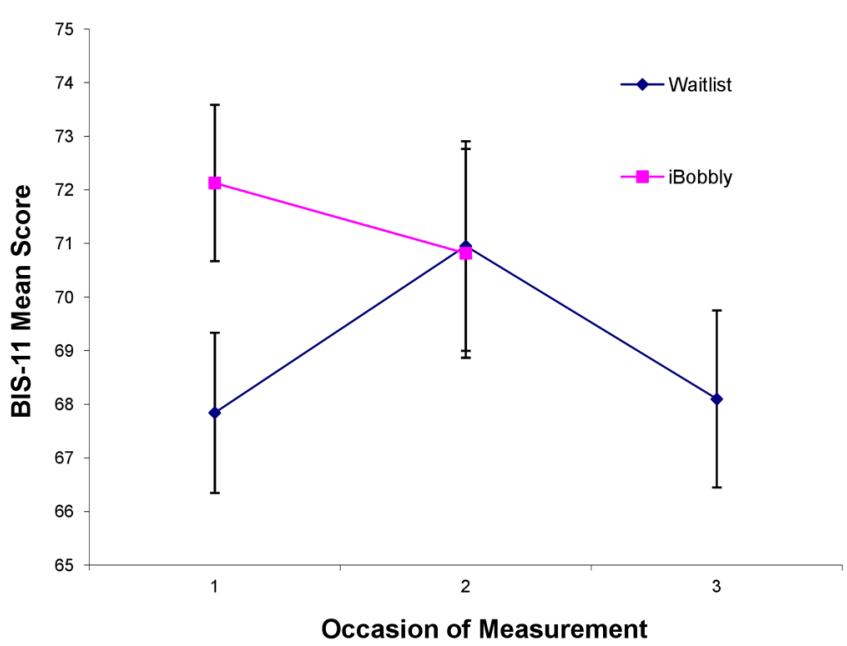

Figure 6 Estimated marginal mean impulsivity (BIS-11) scores by time and intervention arm (error bars represent \pm 1 SE). BIS-11, Barratt Impulsivity Scale.

\section{App usage}

Usage data were available from 40 out of 61 participants. The remaining 21 failed to provide adherence data for a variety of reasons: 3 were lost to follow-up, technical/ connectivity failure accounted for a further 5, and the remaining 13 were either not available to meet in person to download data or had a technical problem with the tablet device such as an uncharged battery. There is the possibility some participants were selfconscious post-trial about sharing this personal usage data or self-conscious about a lack of adherence. Of the 40 participants for whom usage data were available, 34 $(85 \%)$ completed all activities (three self-assessments and three content modules), 1 completed five out of six activities (three self-assessments and two content modules), and 5 completed two out of six activities (one self-assessment and one content module). This shows good adherence to the trial by those whose usage data were available; however, it is also a limitation that we were unable to obtain usage data for 21 participants for the reasons listed above despite only losing 3 participants to follow-up. As a pilot trial and one of the few RCTs using an app and the first with this population, we were also interested in the technical challenges and the feasibility of usage data collection.

\section{DISCUSSION}

The present study found that an app offering acceptance-based therapy reduced depression symptoms and psychological distress in the target population, but did not lead to significant changes on suicidality or impulsivity. Eligibility criteria were modified in response to community need and restricted recruitment area. Thus, 16 of the participants did not have suicide ideation at baseline, making it difficult to demonstrate a significant effect in a small sample. We briefly examined whether change in ideation was present for those with ideation $(n=45)$, but there were no differences found in this subanalysis. Our scales were brief, motivated by the need to make responses non-burdensome, but this may have reduced sensitivity. Since impulsivity measures temperament as opposed to symptoms of distress, our expectation of seeing change may have been too high for this outcome measure. This was supported by the observation of little change in both groups over the time period for this measure, especially in comparison to observed changes in distress and depression.

Nevertheless, the study had strengths. One of the key criticisms of eHealth interventions is that they target those with mild disorders and do not capture those at most risk. In this case, we demonstrated the feasibility and effectiveness of an app for self-help of severe psychological distress. We also demonstrated engagement with an intervention co-designed by community members in one of the most remote regions of the world. The design and execution of the study is also unique, even in the context of rural and urban regions. We are unaware of any other study in any other population which targets suicide ideation using an app and which has been evaluated by an RCT. A 2013 systematic review of evidence-based mobile apps for mental health included only five apps for analysis, ${ }^{27}$ and these did not include suicide ideation. No suicide prevention apps, despite the number publicly available ${ }^{28}$ have been shown to reduce anxiety and depression in the target groups in RCTs. A 2013 systematic review of suicide prevention interventions for Indigenous populations found no RCTs and noted a lack of methodological rigour in the evaluation of Indigenous suicide prevention programmes. $^{29}$

Specifically, the trial demonstrates that eHealth applications may cut through the significant barriers that mental health professionals and researchers find in attempting to improve health outcomes for young Indigenous people. They also demonstrate the value of individual interventions in addition to those targeting the well-being of the community. Nevertheless, the trial required 5 years to complete from initial concept forming, community consultations, through to app development and finally completion of the trial itself. Many Indigenous communities continue to be severely affected by cycles of suicide grief and are often resistant to interventions or research designed by non-Indigenous stakeholders. ${ }^{11}$ As this was the first trial of its type in Indigenous communities, it presented a number of unknowns. It was anticipated that limitations would be reported such as the loss of a large number of participants to follow-up, the loss of tablets or damage incurred to tablets. Therefore, we expected the data obtained at the end of the trial to come from significantly fewer than the 61 registered participants. On the contrary, there was a low attrition rate $(n=2,3 \%)$ which is likely a consequence of the embedded nature of the research within a local partner organisation staffed by Indigenous professionals. The partner organisation had 
pre-existing relationships and a trusted reputation with Indigenous community members. These trusting relationships can feature in Indigenous service provision in remote areas and may be advantageous for future trials. Recruitment processes also indicated the importance of the local partner in the region with $75 \%$ of participants recruited as a result of the promotion of the trial by staff through their networks via word-of-mouth. The willingness of Indigenous staff within the partner organisation and other mental health organisations to promote the research to the community indicated strong acceptance of the research process and the app intervention itself. The willingness of research participants to recommend the app to others in their networks also indicated acceptance and cultural appropriateness of the app for Indigenous youth in the region. A 2016 study that evaluated the acceptability of this app for Indigenous users reported that the app was culturally responsive and potentially an important addition to the public health approach for Indigenous people. ${ }^{30}$ This study highlighted the importance of co-design and suggested the possible inclusion of historical factors that influence Indigenous mental health.

\section{CONCLUSIONS}

This is the first trial of a suicide prevention app in any population group; the first trial to focus on acceptancebased therapies for suicide in Indigenous communities, and the first published RCT of any suicide prevention intervention in an Australian Indigenous community. This trial indicated that an eHealth app developed in partnership with Indigenous communities was accepted and promoted by the target community and improved mental health symptoms.

Twitter Follow Joseph Tighe @joseph_tighe

Acknowledgements This study was funded by the Australian Government Department of Health and Ageing. Samsung Australia generously donated 150 Galaxy tablets for the study. Thoughtworks programmed the app at considerably reduced cost through their partnership with HITnet. The Young and Well Cooperative Research Centre (YAW-CRC) provided a PhD scholarship to examine the effectiveness of the app through this pilot. This study was designed and was carried out with the help of an Indigenous reference group and expert panel members. This group consisted of Indigenous suicide prevention professionals, multimedia professionals, professionals on suicide, depression, web-based interventions or clinical psychology in general. The authors would like to thank research participants, Indigenous community members from the Kimberley region of Western Australia, staff and steering group at Alive and Kicking Goals, Men's Outreach Service, Kimberley based artists, Goolarri Media, Nyamba Buru Yawuru and Yawuru Traditional Owners, Kinway/Anglicare staff in Broome and Derby, BackTrack Armidale, HITnet Innovations, Muru Marri Indigenous Health Unit, University of New South Wales School of Medicine, and the YAW-CRC.

Collaborators JT made a substantial contribution to the study design, Indigenous community partnerships management, recruitment, data interpretation, statistical analyses and writing of this manuscript. FS and $\mathrm{HC}$ made a substantial contribution to the study design, data interpretation and writing. RR assisted with several elements of the study design. She was responsible for garnering ethics approval across several committees, as well as setting up online data managements systems. RR provided the project management for the creation of the app which included consolidating text, graphics and voice overs content. She also assisted in the editing of this manuscript. AM made a substantial contribution to the trial design, statistical analyses, production of figures, interpretation of the data, writing the manuscript and critically reviewing the manuscript. NDLM assisted with the analysis plan of the data and ensured the appropriate statistical methods were selected and validated statistical analyses. NDLM provided feedback on the draft manuscripts and approved of the final manuscript.

Funding The trial was funded by the Australian Government Department of Health and Ageing.

Competing interests None declared.

Ethics approval University of New South Wales Human Ethics Committee (Ref:HC13025), the Western Australian Aboriginal Health Ethics Committee (Ref:486) and the Kimberley Aboriginal Health Planning Forum (Ref:2013-006).

Provenance and peer review Not commissioned; externally peer reviewed.

Data sharing statement Additional data can be accessed via the Dryad data repository at http://datadryad.org/ with the doi:10.5061/dryad.860kn.

Open Access This is an Open Access article distributed in accordance with the Creative Commons Attribution Non Commercial (CC BY-NC 4.0) license, which permits others to distribute, remix, adapt, build upon this work noncommercially, and license their derivative works on different terms, provided the original work is properly cited and the use is non-commercial. See: http:// creativecommons.org/licenses/by-nc/4.0/

\section{REFERENCES}

1. AlH. Overview of Australian Indigenous health status. 2014 Retrieved 25 Oct 16 from http://www.healthinfonet.ecu.edu.au/ health-facts/overviews.

2. Statistics ABS. Aboriginal and Torres Strait Islander suicide deaths 2012. Retrieved 25 Oct 16 from http://www.abs.gov.au/ausstats/ abs@.nsf/Products/3309.0 2010 Chapter Aboriginal+and+Torres +Strait+Islander+suicide+deaths?OpenDocument

3. Hunter E. Aboriginal health and history: power and prejudice in remote. Australia: Cambridge University Press, 1993.

4. Tatz C. Aboriginal suicide is different: A portrait of life and selfdestruction. Canberra, Australia: Aboriginal Studies Press 2005:xxv.

5. Hunter E, Milroy H. Aboriginal and Torres Strait Islander suicide in context. Arch Suicide Res 2006;10:141-57.

6. Dudgeon P, Walker R. Decolonising Australian psychology: discourses, strategies, and practice. J Soc Political Psychol 2015;3:276-97.

7. Gracey M, King M. Indigenous health part 1: determinants and disease patterns. Lancet 2009;374:65-75.

8. Isaacs AN, Pyett P, Oakley-Browne MA, et al. Barriers and facilitators to the utilization of adult mental health services by Australia's Indigenous people: seeking a way forward. Int J Ment Health Nurs 2010;19:75-82.

9. National Mental Health Commission. Contributing Lives, Thriving Communities - Report on the National Review of Mental Health Programmes and Services. Sydney: NMHC. 2014: Fact Sheet 2 - What this means for Aboriginal and Torres Strait Islander people.

10. Aboriginal Health Organisation of South Australia, Clayer JR. Mental health and behavioural problems in the urban Aboriginal population. South Australian Health Commission; 1991.

11. Dudgeon PWR, Scrine C, Cox K, et al. Enhancing wellbeing, empowerment, healing and leadership. Chapter 25. In: Dudgeon $P$ Milroy $\mathrm{H}$, Walker R, eds. Working together: Aboriginal and Torres Strait Islander mental health and wellbeing principles and practice 2014. 2nd edn. 2014:437-48.

12. Chandler M, Lalonde C. Cultural continuity as a moderator of suicide risk among Canada's First Nations. In: Kirmayer LJ, Valaskakis GG, eds. Healing traditions: the mental health of Aboriginal peoples in Canada. Vancouver: University of British Columbia Press, 2009.

13. Ridani $\mathrm{R}$, Shand FL, Christensen $\mathrm{H}$, et al. Suicide prevention in Australian aboriginal communities: a review of past and present programs. Suicide Life Threat Behav 2015;45:111-40.

14. Research MI. Media Usage amongst Aboriginal and Torres Strait Islander People. 2014. Retrieved 13 Oct 16 from http://mcnair. com.au/media-release-media-usage-amongst-aboriginal-andtorres-strait-islander-people-infographic/ 
15. Hayes SC. Acceptance and commitment therapy, relational frame theory, and the third wave of behavioral and cognitive therapies. Behav Ther 2004;35:639-65.

16. Shand FL, Ridani R, Tighe J, et al. The effectiveness of a suicide prevention app for indigenous Australian youths: study protocol for a randomized controlled trial. Trials 2013;14:396.

17. Tighe J, McKay K. Alive and kicking goals!: preliminary findings from a Kimberley suicide prevention program. Adv Mental Health 2012;10:240-5.

18. Metalsky GI, Joiner TE. The hopelessness depression symptom questionnaire. Cogn Ther Res 1997;21:359-84.

19. Joiner TE, Rudd MD. Negative attributional style for interpersonal events and the occurrence of severe interpersonal disruptions as predictors of self-reported suicidal ideation. Suicide Life Threat Behav 1995;25:297-304.

20. Joiner TE, Rudd MD. Disentangling the interrelations between hopelessness, loneliness, and suicidal ideation. Suicide Life Threat Behav 1996;26:19-26.

21. Joiner TE, Pfaff JJ, Acres JG. A brief screening tool for suicidal symptoms in adolescents and young adults in general health settings: reliability and validity data from the Australian National General Practice Youth suicide prevention project. Behav Res Ther 2002;40:471-81.

22. Spitzer RL, Williams JBW, Kroenke K. Instructions for Patient Health Questionnaire (PHQ) and GAD-7 measures. (n.d.)
Retrieved 12 Jan 2017 from http://www.phqscreeners.com/ overview.aspx.

23. Kessler RC, Barker PR, Colpe LJ, et al. Screening for serious mental illness in the general population. Arch Gen Psychiatry 2003;60:184-9.

24. Andrews $\mathrm{G}$, Slade T. Interpreting scores on the Kessler psychological distress scale (K10). Aust N Z J Public Health 2001;25:494-7.

25. Patton $\mathrm{JH}$, Stanford MS, Barratt ES. Factor structure of the Barratt impulsiveness scale. J Clin Psychol 1995;51:768-74.

26. Thornicroft G, Beecham J, Knapp M. Costing psychiatric interventions. Measuring Mental Health Needs London: Gaskell 2001:220-4.

27. Donker T, Petrie K, Proudfoot J, et al. Smartphones for smarter delivery of mental health programs: a systematic review. J Med Internet Res 2013;15:e247.

28. Larsen ME, Nicholas J, Christensen H. A Systematic assessment of smartphone tools for suicide prevention. PLOS ONE 2016;11: e0152285.

29. Clifford AC, Doran CM, Tsey K. A systematic review of suicide prevention interventions targeting indigenous peoples in Australia, United States, Canada and New Zealand. BMC Public Health 2013;13:463.

30. Povey J, Mills PPJR, Dingwall KM, et al. Acceptability of mental health apps for aboriginal and torres strait islander Australians: a qualitative study. J Med Internet Res 2016;18(3):e65:1-12. 